\title{
ESTUDIO BIBLIOMETRICO SOBRE LA PRODUCCIÓN CIENTÍFICA BRASILEÑA EN REVISTAS ESPAÑOLAS DURANTE EL PERÍODO DE 1997 A 2000¹
}

\author{
Vanda Ferreira dos Santos
}

\section{Título}

Estudo bibliométrico sobre a produção científica brasileira em revistas espanholas do período de 1997 a 2000

\section{Resumo}

Estudo de bibliométrico sobre a produção científica brasileira em revistas espanholas, com o objetivo para avaliar a produção científica de autores brasileiros na Espanha. A análise foi feita através de artigos científicos publicados em revistas espanholas coletadas pelas bases de dados de Ciência e Tecnologi'a (ICYT) e de Ciências Sociais e Humanidades (ISOC) do Conselho Ssuperior de Investigações Científicas (CSIC). Os resultados da investigação mostram que a contribuição brasileira ao conjunto das bases ICYT e ISOC é mínima, tal afirmativa baseia-se na participação brasileira nessas bases de dados: em Ciência e Tecnologia não representou $1 \%$ e em Ciências Sociais e Humanindades e não alcançou $0.50 \%$ dos trabalhos recolhidos.

\section{Palavras-Chave}

Bibliometria; Produção Científica; Revistas Espanholas

\footnotetext{
${ }^{1}$ Trabajo de conclusión de la asignatura "Indicadores para Evaluar la Ciencia y la Tecnologia" del Doctorado en Documentación de la Universidad Carlos III de Madrid.
} 


\section{INTRODUCCIÓN}

Las revistas científico-técnicas son uno de los canales más utilizados para la difusión de los resultados de investigación. A través de los artículos de revistas, el investigador expone sus ideas, garantiza la propiedad intelectual y se somete a la evaluación de los pares, principalmente, cuando se consideran aquellas revistas en las que existe un sistema de selección que garantiza la calidad y originalidad de los trabajos. En consecuencia, el resultado de las investigaciones científicas contribuye al avance de la ciencia. Esa actividad de diseminación produce un flujo informativo que propicia la transformación de la información en conocimiento.

La producción científica y tecnológica de un país está determinada por los recursos económicos que el gobierno destina para las actividades de investigación y desarrollo (I+D) y por la inversión en capital humano investigador. Uno de los factores que han impulsados el progreso de la ciencia y de la técnica en la actualidad han y ha sido precisamente el intercambio de informaciones.

Según Pirró E Longo (1996, apud TARGINO; GARCIA, 2000) Brasil ha seguido la tendencia mundial a partir de la Segunda Guerra Mundial de invertir en ciencia y tecnología como actores claves para su progreso. El Segundo Plan Nacio- nal de Desarrollo - II PND (1975-79) agilizó la estructuración de un sistema de ciencia y tecnología y tuvo como objetivo principal la institucionalización de los cursos de postgrado en Brasil. Los resultados de toda esta inversión reflejan que Brasil está entre los diez mayores del mundo, su Producto Interno Bruto (PIB) es de US\$ 735.301 billones, según datos del Instituto Brasileño de Geografía y Estadística (IBGE).

Según Martín Sempere (1991) en su estudio sobre la producción científica y tecnológica de los países latinoamericanos en la base de datos ICYT, del Consejo Superior de Investigaciones Científicas CSIC, de España, sólo 19 países están representados en la citada base, con sólo el 2,6\%. En este trabajo Brasil es el sexto país de mayor producción en las revistas españolas, después de Argentina, Chile, Cuba, Venezuela y México con una aportación del 0,10\%. Por otro lado, según Diez Hoyo (1993), Brasil está en primer lugar en el rango de los países latinoamericanos que publican en Europa con una aportación del $10,5 \%$.

El presente estudio tiene como objetivo evaluar la producción científica de autores brasileños en España, a través del análisis de los artículos científicos publicados en revistas españolas recogidas en las bases de datos de Ciencia y Tecnología ICYT y de Ciencias Sociales y Humanidades - ISOC del CSIC. 


\section{OBJETIVOS}

El análisis de las publicaciones permitirá conocer los siguientes aspectos:

- Número de documentos procedentes de autores cuya afiliación institucional esté radicada en Brasil;

- Participación relativa de estos autores en el conjunto de las bases de datos ICYT y ISOC;

- Temática abordada;

- Idioma de los trabajos;

- Tipo de institución con mayor participación en la realización de los trabajos;

- La(s) región(es) brasileña(s) de mayor representatividad en los trabajos;

- Trabajos realizados en colaboración nacional;

- Trabajos realizados en colaboración internacional.

\section{METODOLOGÍA}

Para recoger la información contenida en las publicaciones, se han consultado dos bases de datos: ISOC e ICYT del CSIC. Las bases de datos del CSIC recogen referencias bibliográficas de artículos publicados en más de 2.000 revistas editadas en España en Ciencias Sociales y Humanidades y en Ciencia y Tecnología. La base ISOC recoge los artículos publicados en más de 1.625 revistas científicas españolas y, otros documentos (informes técnicos, comunicaciones a congresos, monografías, etc.). En la actualidad reúnen más de 383.000 referencias bibliográficas, con un crecimiento anual de 25.000 referencias y una actualización diaria. Aunque de algunas materias se recoge información desde 1960 , más del $50 \%$ de la base corresponde a literatura publicada en los últimos 10 años. La base ICYT recoge 500 publicaciones periódicas especializadas editadas en España, fundamentalmente revistas, además anuarios, memorias, monografías, actas de congresos y tesinas. La base ICYT cubre un período de 1979 hasta el presente. Actualmente contiene 135.000 referencias, con un incremento de 8.000 referencias anuales.

A partir de estas bases de datos, y con el fin de analizar los artículos publicados en los últimos 4 años $(1997 / 2000)^{2}$ se han elaborado dos bases de datos, en Excel 97 filtrado, a partir del resultado de la búsqueda en dichas bases, denominadas ISOC/BR y ICYT/BR.

La clasificación de las instituciones de trabajo de los autores fue según su repetición en la base, siendo éstas: para el área de Ciencias Sociales y Humanidades: Universidad, Institutos de Investigación, Fundación, Empresas, Otros y Sin Informar. Para el área de Ciencia y Tecnología: Uni-

\footnotetext{
${ }^{2}$ Estos años corresponden a la fecha de publicación de las revistas
} 
versidad, Centros de Investigación, Laboratorios, Hospitales, Empresas, Otros y por último, Sin informar. Las regiones brasileñas fueron clasificadas según su distribución geográfica, siendo éstas: Sudoeste, Nordeste, Norte, Sur y CentroOeste.

\section{RESULTADOS Y DISCUSIÓN}

\subsection{Producción científica}

A) Ciencia y Tecnología

El número de documentos correspondiente a trabajos de autores brasileños en la Base ICYT durante los años 1997-2000 son respectivamente $39,69,70$ y 58 . En la Tabla 1 se presenta la participación relativa de estos trabajos en el conjunto de la base de datos.

El número total de documentos contenidos en la base ICYT en el año 1997, es de 7897 , de ellos, 39 corresponden a tra- bajos de autores brasileños que han sido publicados en las revistas científicotécnicas españolas, la aportación científica brasileña al total de documentos de la base, en el año, es de 0,49\%. En el año 1998 su participación aumentó alcanzando el $0,87 \%$ del total de documentos de la base. En el año 1999 se mantuvo el crecimiento llegando al $0,91 \%$ del total. El año 2000 hubo un descenso respecto a los años previos, alcanzando el $0,83 \%$ del total de documentos de la base.

Del conjunto de documentos de la base ICYT durante los 4 años, la producción científica brasileña alcanza el $0,77 \%$. Se observa que la producción anual registrada en 1999 se ha duplicado prácticamente en relación con el año 1997, llegando al $0,91 \%$. Asimismo puede verse que la aportación brasileña no alcanza el $1 \%$ del total de la base.

Tabla 1 Distribución cronológica del número de trabajos en la base ICYT

\begin{tabular}{c|c|c|c}
\hline Año & Doc. Brasileños & Total de La Base ICYT & $\%$ \\
\hline 1997 & 39 & 7897 & 0,49 \\
\hline 1998 & 69 & 7965 & 0,87 \\
\hline 1999 & 70 & 7658 & 0,91 \\
\hline 2000 & 58 & 6969 & 0,83 \\
\hline total & 236 & 30489 & 0,77 \\
\hline
\end{tabular}

B) Ciencias Sociales y Humanidades

El número de documentos correspondiente a trabajos de autores brasileños en 
la base ISOC durante los años 1997-2000 son respectivamente $55,75,59$ y 33 . En la Tabla 2 se presenta el porcentaje de estos trabajos en el total de la base ISOC.

En el año 1997, el número total de documentos contenidos en la base ISOC, es de 20586, de ellos, 55 corresponden a trabajos de autores brasileños. La aportación brasileña al total de documentos de la base en este año es de $0,27 \%$. En el año 1998 hubo un crecimiento, representando el $0,38 \%$ del total de documentos. El año 1999 hubo un descenso, alcanzando el
0,32\% y en el año 2000 se mantuvo el decrecimiento llegando al $0,25 \%$ del total de documentos de la base. Del Conjunto de documentos de la base ISOC durante los 4 años, la producción científica brasileña alcanza el 0,31\%. En la Tabla 2 puede observarse que en el período analizado la cantidad de trabajos de autores brasileños se mantuvo, el crecimiento que hubo entre el año 1997/98 no se repitió en los años posteriores. La aportación científica brasileña en la base ISOC no llega al $0,50 \%$ del total de la base.

Tabla 2 Distribución cronológica del número de trabajos en la base ISOC

\begin{tabular}{c|c|c|c}
\hline Año & Doc. Brasileños & Total de La Base ISOC & $\%$ \\
\hline 1997 & 55 & 20586 & 0,27 \\
\hline 1998 & 75 & 19738 & 0,38 \\
\hline 1999 & 59 & 18355 & 0,32 \\
\hline 2000 & 33 & 13086 & 0,25 \\
\hline total & 222 & 71765 & 0,31 \\
\hline
\end{tabular}

Tratando de establecer una comparación entre los resultados se puede decir que la aportación científica brasileña en España es mayor en el área de Ciencia y Tecnología que en el área de Ciencia Sociales y Humanidades. Pero también habrá que considerar que el volumen total de la base en Ciencia Sociales y Humanidades es mayor que en Ciencia y Tecnología.

\subsection{Temática abordada}
A) Ciencia y Tecnología

La cantidad de documentos recuperados en el área de Ciencia y Tecnología fueron de 236 trabajos.

B) Ciencias Sociales y Humanidades

La cantidad de documentos recuperados en el área de Ciencias Sociales y Humanidades fueron de 222 trabajos.

Con los resultados se puede decir que hubo una producción relativa mayor en 
el área de Ciencia y Tecnología que en el área de Ciencia Sociales y Humanidades.

\subsection{Idioma de los trabajos}
A) Ciencia y Tecnología

En el Gráfico 1 se representa el idioma utilizado por los autores en la publicación de sus resultados de investigación.

\section{Gráfico 1}

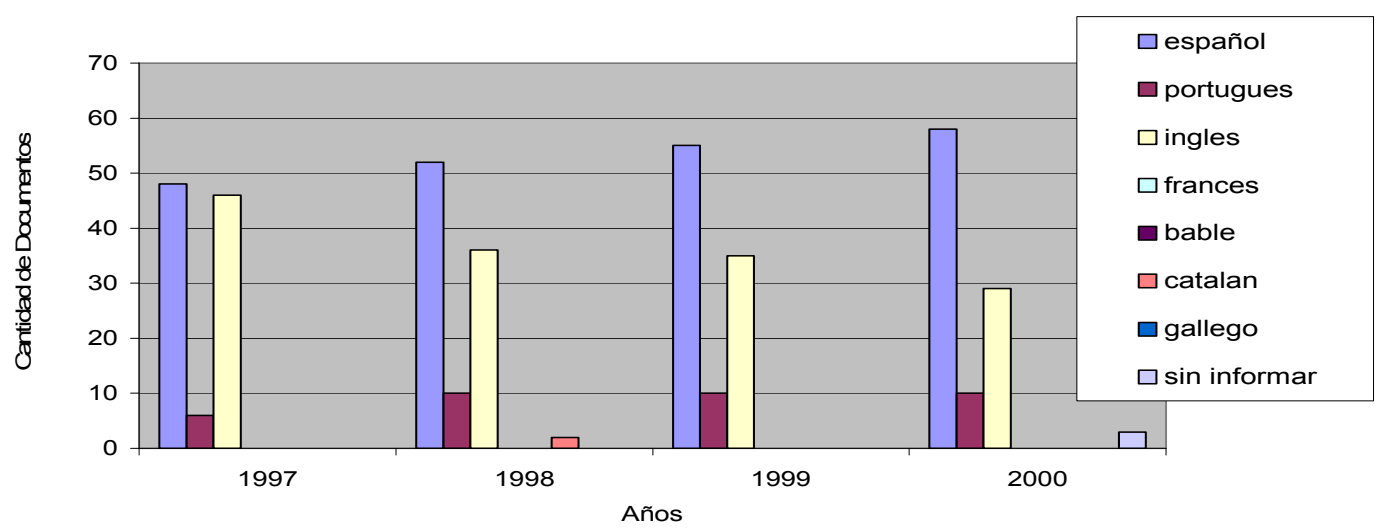

El idioma español es el más utilizado con una media de $53 \%$ durante el período analizado. El idioma inglés es el segundo más representativo con una media del $37 \%$. El portugués ocupa el tercer lugar ya que no llega a representar el $15 \%$ de los trabajos publicados. Obtuvo una media del $10 \%$ de los documentos durante los años estudiados. Cabe destacar que se ha encontrado sólo un trabajo en catalán. Otro punto a subrayar es que los trabajos en español tuvieron una tendencia de crecimiento en el período en cuanto los trabajos en inglés tuvieron una tendencia de a la baja en los años estudiados.

B) Ciencias Sociales y Humanidades
En el Gráfico 2 se representa el idioma de los trabajos, en él puede verse que el idioma español es lo más representativo, con el $80 \%$ durante en los años estudiados. El idioma portugués se presenta en segundo lugar representando el $16 \%$ y el inglés es el tercero, pero no llegó a tener una gran representación, quedándose con el 2,3\% en los años analizados. Cabe subrayar que se han encontrado trabajos en idiomas como el francés, catalán, gallego y bable, los trabajos en estos idiomas no llegaron a superar el $2 \%$. Otro punto a destacar es que los trabajos en español disminuyeron en el período en cuanto los trabajos en portugués tuvieron una ligera tendencia de crecimiento en los años estudiados. 


\section{Gráfico 2}

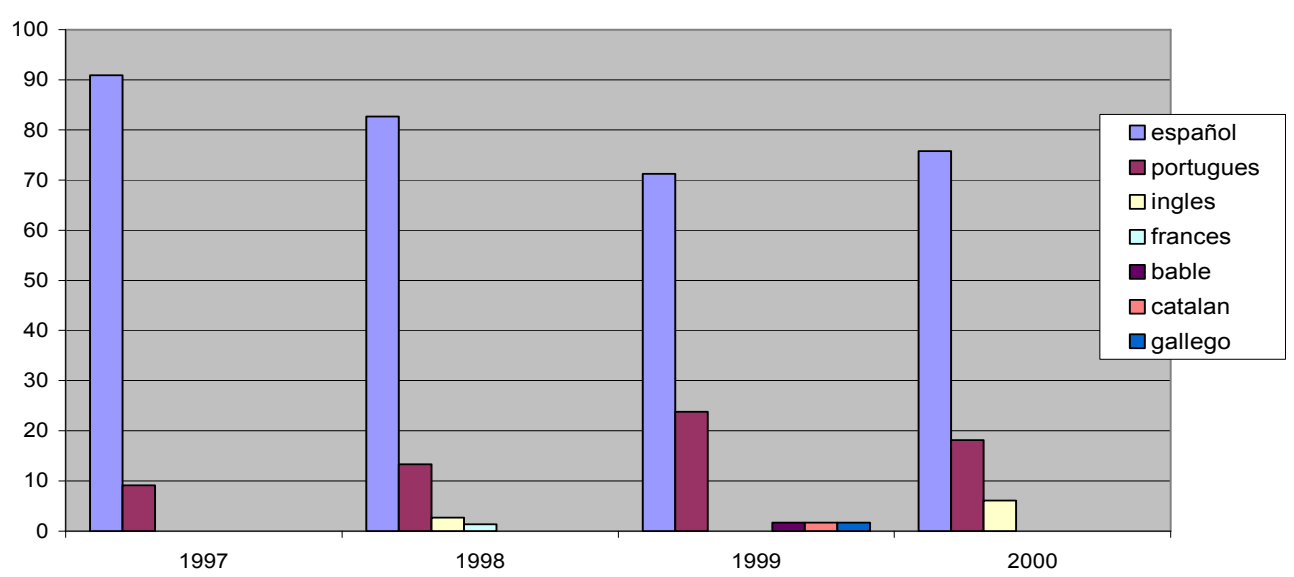

Estableciendo una comparación entre los idiomas de los trabajos en Ciencia y Tecnología y Ciencia Sociales y Humanidades se puede decir que hay una tendencia distinta en los idiomas de los trabajos. El inglés es más utilizado en el área de las Ciencias Puras, Básicas y Experimentales que en las Ciencias Sociales. Hubo una tendencia de uso del inglés en los trabajos del área de Ciencia y Tecnología y del portugués en los trabajos de Ciencia Sociales y Humanidades.

\subsection{Tipo de Institución}

El número de instituciones es mayor que el número de documentos recuperados debido a la existencia de trabajos en colaboración, y, por tanto, en algunos casos, para un único trabajo se ha contabilizado más de una institución. No se han considerado las colaboraciones intrainstitucional, es decir los trabajos en colaboración entre Departamentos o Facultades de una misma institución.

A) Sectores institucionales en las áreas de $\mathrm{C}$ y $\mathrm{T}$

En el Gráfico 3 se representa la distribución de las instituciones de trabajo de los autores en el área de Ciencia y Tecnología, en él puede verse que la Universidad es la institución más representada, con el 63\%, $74 \%, 78 \%$ y $77 \%$ respectivamente en los años analizados. 


\section{Gráfico 3}

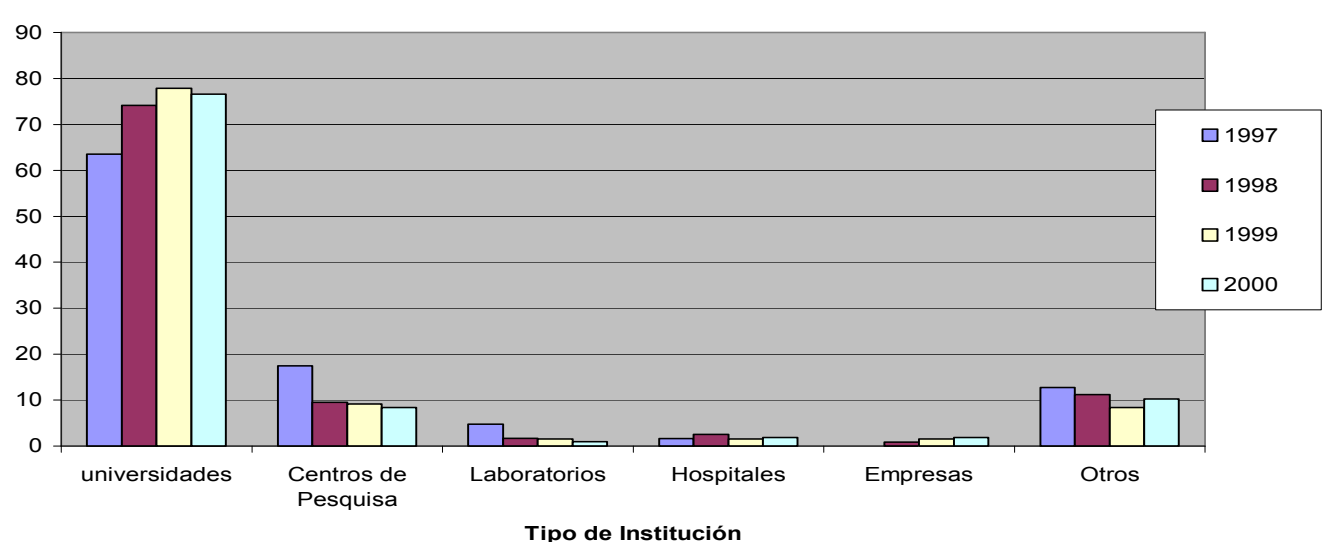

Se recuperaron trabajos realizados en Universidades de gran prestigio en Brasil, tales como son la Universidad de Sao Paulo (USP), Universidad Federal del Río de Janeiro (UFRJ), Universidad de Brasilia (UNB), Universidad Federal de Bahía (UFBA), Universidad Federal de Ceará (UFC), Universidad Federal de Pernambuco (UFPE), Universidad Federal del Río Grande del Sur (UFRGS) entre otras. En segundo lugar en el año 1997 aparecen los Centros de Investigación con el destaque de la Empresa Brasileña de Investigación Agropecuaria (EMBRAPA) y sus Centros Especializados, tales como el Centro de Investigación Agropecuaria del Trópico Semiárido (CPATSA), Centro Nacional de Investigación de Tecnología de Agroindustria Alimentar (CTAA), Centro Nacional de Investigación Mandioca y Fruticultura (CNPMF), entre otros. En el año 1998 el segundo lugar con Otros representando los trabajos realizados por instituciones como Fundación Oswaldo Cruz (FIOCRUZ), Consejo Superior de Investigaciones Científicas (CSIC), entre otros. En el año 1999 los Centros de Investigación volvieron al segundo lugar en el rango de las instituciones. En el año 2000 Otros volvió al segundo lugar en el rango. El tercer lugar fue asumido por Otros en los años 1997/1999 y por los Centros de Investigación en los años 1998 y 2000. Los últimos lugares fueran ocupados por los Laboratorios, destacándose el Laboratorio Nacional de Computación del Río de Janeiro, los Hospitales, tales como, la Santa Casa del Río Grande del Sur; y las Empresas, como: Sularroz Benef. Comerc. Arroz, El DuPont de Nemours y la Empresa Pernambucana de Pesca Agropecuaria. 
Cabe señalar que la mayoría de los trabajos fueron realizados en la universidad o en colaboración con ella, excepto algunos trabajos realizados por empresas, centros de investigación o hospitales que no contaron con la participación de la universidad.
B) Sectores institucionales en las áreas de Ciencias Sociales y Humanidades

En el Gráfico 4 se presenta la distribución de las instituciones de trabajo de los autores en el área de Ciencia Sociales y Humanidades.

\section{Gráfico 4}

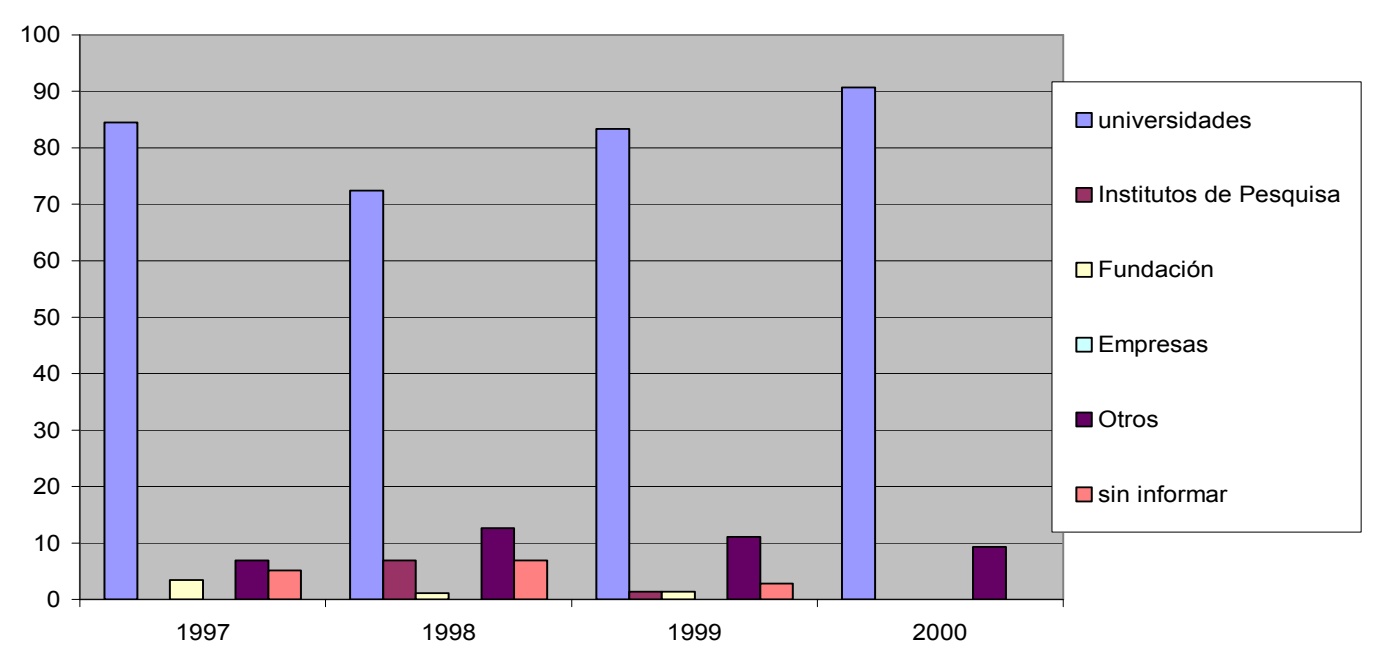

Es visible que la Universidad es la institución con el mayor número de trabajos presentados con un $84,72,83$ y $91 \%$ del total de documentos recuperados en los años estudiados. Se recuperaron trabajos realizados en las universidades brasileñas de gran prestigio con destaque para las universidades federales y estatales. Otros, figura en el segundo lugar, en él se ha englobado aquellos organismos que no corresponden a ninguna de las instituciones clasificadas anteriormente en la distribución, tales como: Museos, Archivos Públicos,
Embajada, Oficina Comercial, la Fiscalía, entre otros. Cabe destacar que la ausencia de información (sin informar) de la institución al cuál pertenece el autor figura en tercer lugar, seguido por los Institutos de Investigación y Fundaciones. No hubo autores pertenecientes a una empresa.

Estableciendo una comparación entre las instituciones de trabajo de los autores en el área de Ciencia y Tecnología, y Ciencia Sociales y Humanidades, se puede decir que ambas áreas se coinciden el porcentaje de participación de las institucio- 
nes, lo que difiere en el área de Ciencia Sociales y Humanidades son las instituciones que fueron consideradas como "Otros" que tienen rasgos distintos de aquellas que fueron clasificadas en Ciencia y Tecnología.

\subsection{Distribución por región}

El número de trabajos por región es mayor que el número de documentos recu- perados debido a la existencia de trabajos en colaboración, y, por tanto, en algunos casos, para un único trabajo se ha contabilizado más de una región.

\section{A) Ciencia y Tecnología}

La región de mayor concentración de trabajos en la base es la Sudoeste (Gráfico 5) alcanzando el 67\%, 64\%, 64\% y 55\% respectivamente en los años estudiados.

\section{Gráfico 5}

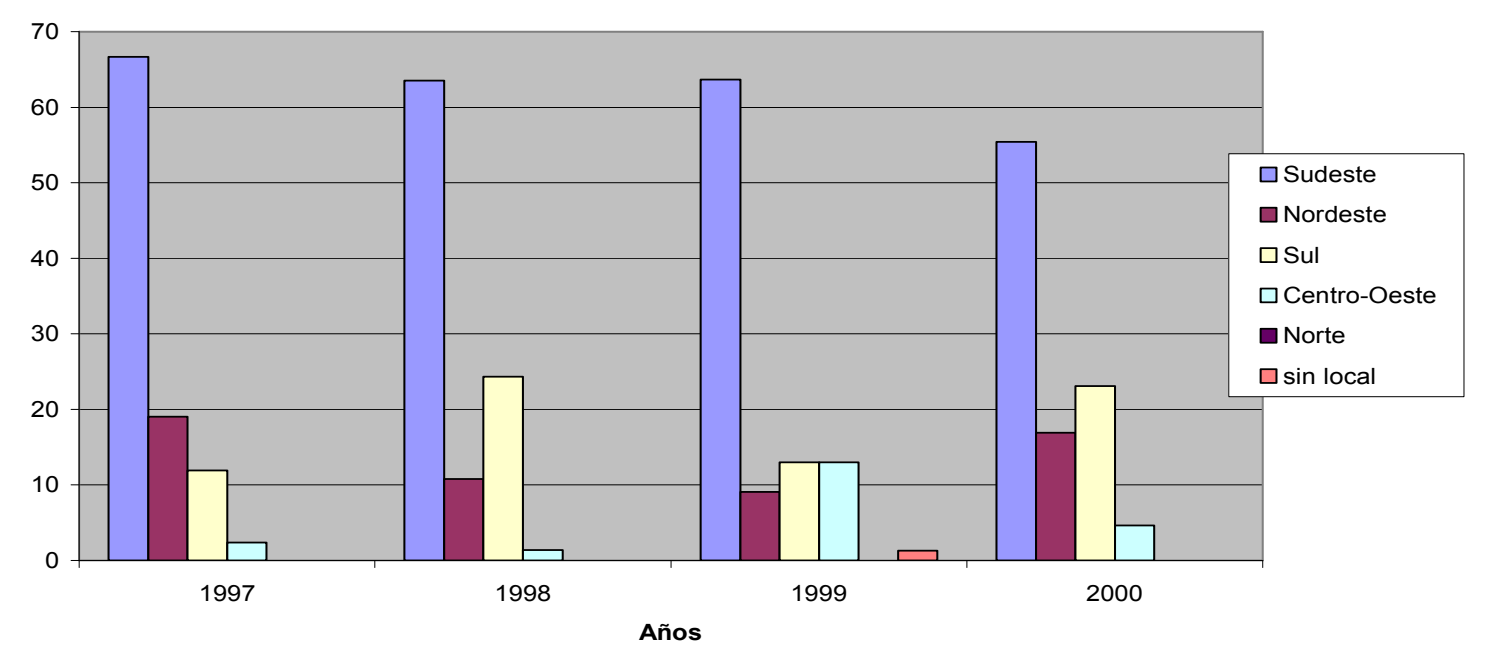

En el año 1997 la región Nordeste se quedó en segundo lugar (19\%), lo mismo sucedió en los años posteriores, donde la región Sur ocupó el segundo lugar $(24 \%$ y $23 \%$ ) en los años 1998 y 2000, en el año 1999 la región Sur compartió con la Centro-Oeste el segundo lugar. No hubo trabajos de la región Norte. Cabe destacar que la región Sudoeste tuvo una tendencia de decrecimiento en los años estudiados no presentado por las otras regiones.
Los estados más representativos por región son: en la región Sudoeste: Río de Janeiro y Sao Paulo; en la región Sur: Río Grande del Sur y Paraná; en la región Nordeste: Bahía, Pernambuco y Ceará; en la región Centro-Oeste: Brasilia.

B) Ciencias Sociales y Humanidades

La región con mayor número de trabajos en la base es la Sudoeste (Gráfico $6)$, representando el 58, 51, 56 y $39 \%$ res- 
pectivamente de los documentos recupe- rados.

\section{Gráfico 6}

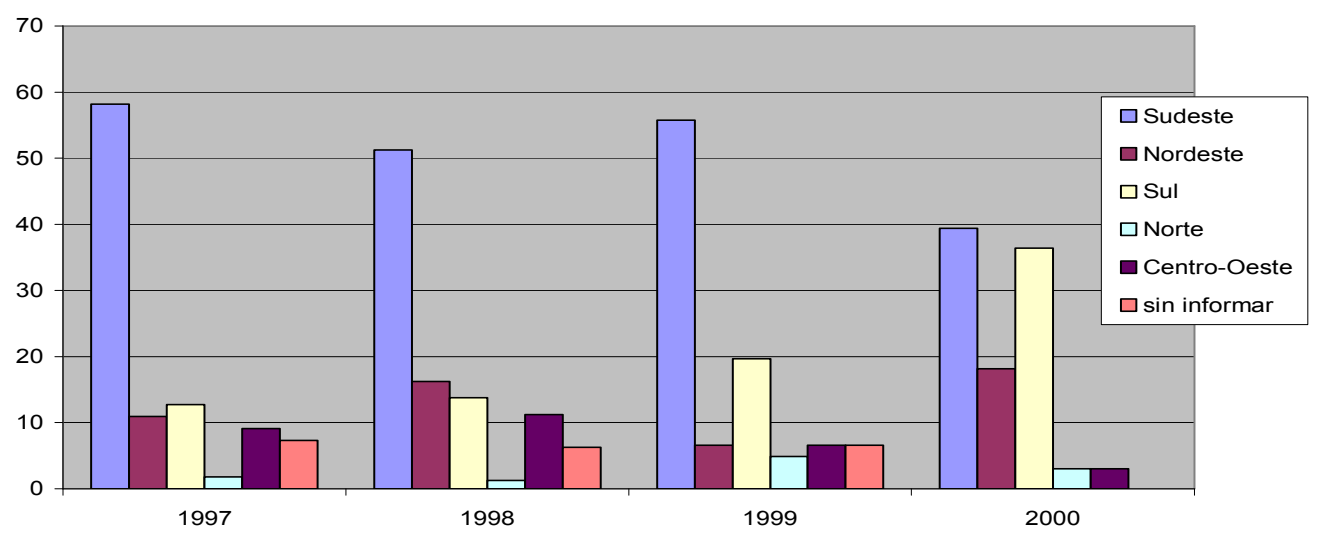

La región con mayor número de trabajos en la base es la Sudoeste (Gráfico $6)$, representando el $58,51,56$ y $39 \%$ respectivamente de los documentos recuperados. En segunda posición en los años 1997, 1999 y 2000 la región Sur. En el año de 1998 la región Nordeste ocupó la segunda posición, representando el $16 \%$ de los trabajos recuperados. La región CentroOeste ocupó la cuarta posición con 7\%, $11 \%, 6 \%$ y $3 \%$ y la región Norte alcanzo el $5 \%$ en el año 1999, ocupando el ultimo lugar en el rango de regiones. Cabe destacar que la región Sur fue la única que mantuvo una tendencia de crecimiento en los años estudiados, las otras regiones no mantuvieron la misma característica.

Tratando de establecer una comparación entre la representación de las regiones de los trabajos en Ciencia y Tecnología y Ciencia Sociales y Humanidades se puede decir que hubo una tendencia similar de representación de las regiones en ambas áreas, con excepción para la región Norte que aportó trabajos solamente en el área de Ciencia Sociales y Humanidades.

\subsection{Colaboración}

A) Ciencia y Tecnología

En el Gráfico 7 se presenta el porcentaje de colaboración, en ella puede verse que los trabajos en colaboración entre 20 más instituciones representan el 82, 91, 83 y $89 \%$ respectivamente de los documentos recuperados. Los trabajos en colaboración nacional representan el 35\%, 41\%, 40\%, y $49 \%$ respectivamente en los años estudiados. Los trabajos en colaboración internacional representan el respectivamente $65 \%, 59 \%, 60 \%$ y $51 \%$ del total de los documentos en colaboración. 


\section{Gráfico 7}

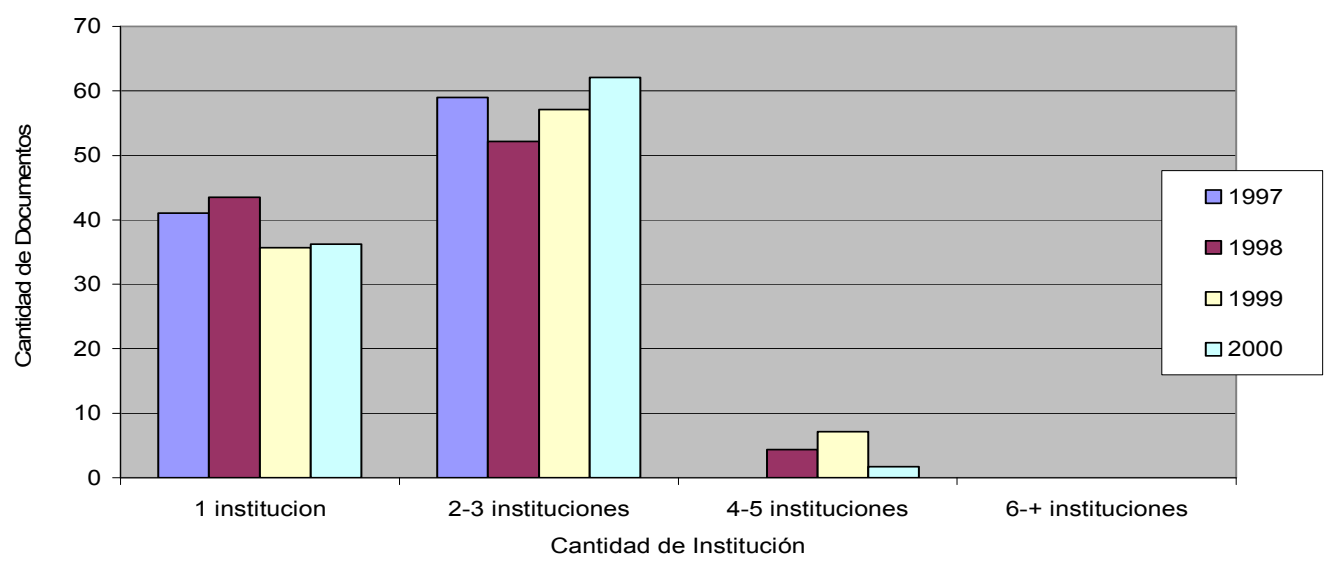

Los resultados reflejan la tendencia de colaboración en el área de Ciencia y Tecnología, especialmente, la internacional que alcanzo índices más representativos que la nacional. España ha sido el país que más ha realizado trabajos en colaboración con Brasil representando los $57 \%$ de las colaboraciones internacionales, este número es elevado y justificable, pues se trata de revistas españolas. Argentina es el segundo país con $11 \%$. En la tabla 3 se detalla la distribución de estas colaboraciones. En el Gráfico 8 se presenta el índice de coautoría en el área de Ciencia y Tecnología.

Tabla 3 Distribución de las cooperaciones internacionales en Brasil

\begin{tabular}{l|c|c}
\hline \multicolumn{1}{c|}{ Países } & Cantidad & $\%$ \\
\hline Alemania & 1 & 1 \\
\hline Argentina & 11 & 11 \\
\hline Canadá & 2 & 2 \\
\hline Chile & 3 & 3 \\
\hline Cuba & 4 & 4 \\
\hline España & 57 & 57 \\
\hline Estados Unidos & 6 & 6 \\
\hline Francia & 2 & 2 \\
\hline México & 2 & 2 \\
\hline Nueva Zelanda & 1 & 1 \\
\hline Reino Unido & 7 & 7 \\
\hline Suecia & 1 & 1 \\
\hline Uruguay & 3 & 3 \\
\hline \multicolumn{1}{c}{ Total } & 100 & 100 \\
\hline
\end{tabular}




\section{Gráfico 8}

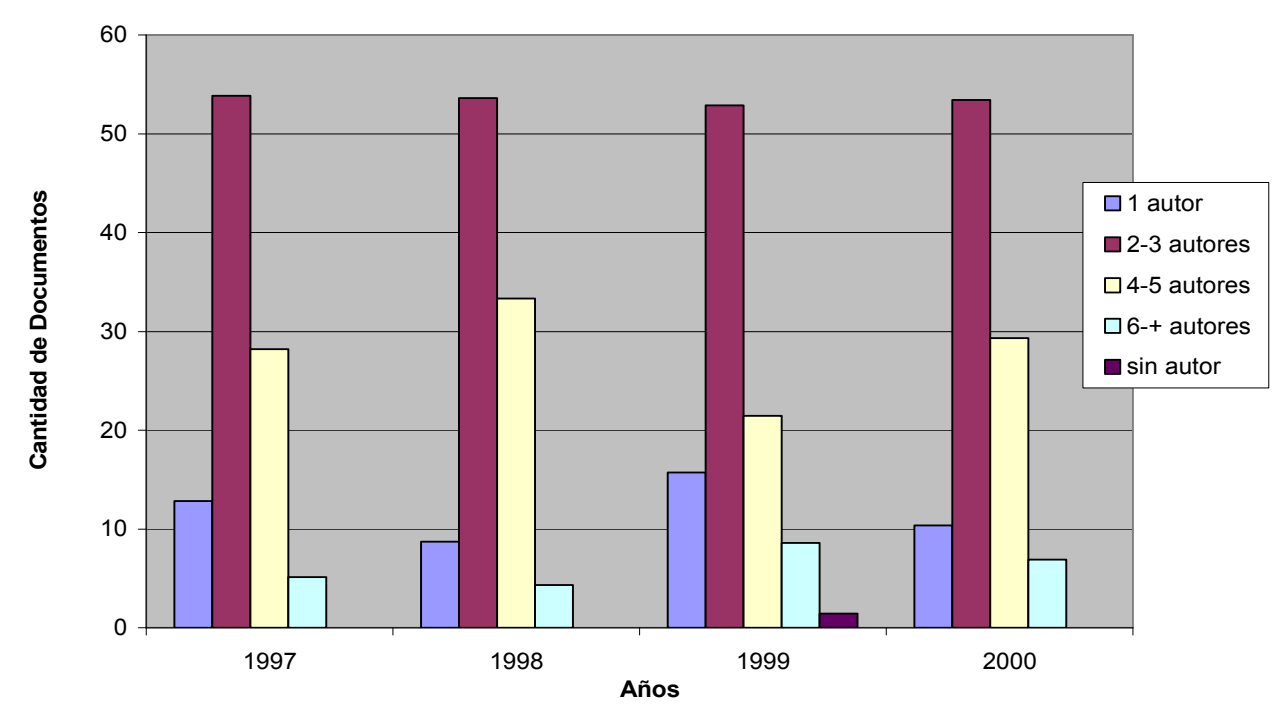

B) Ciencias Sociales y Humanidades

los trabajos hechos en colaboración representan el $5 \%, 15 \%, 10 \%$ y $9 \%$ respectiva-

En Gráfico 9 se presenta el porcenta- mente del total de documentos recuperaje de colaboración, en ella puede verse que dos en los años analizados.

\section{Gráfico 9}

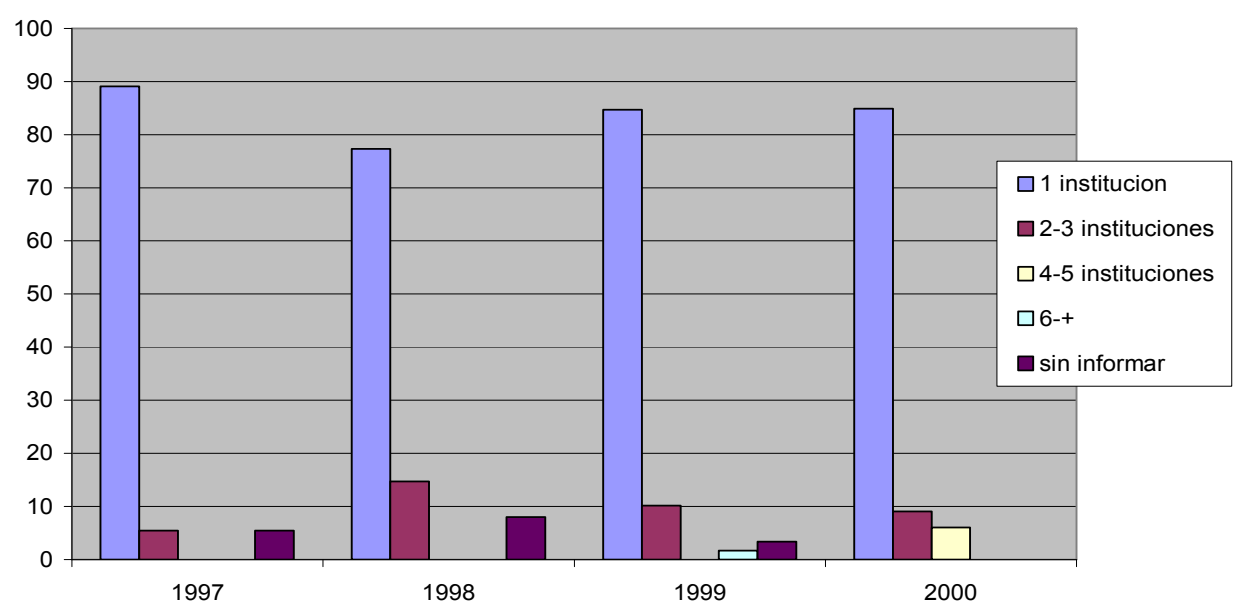

En el año de 1997 los trabajos en colaboración internacional fueron en mayor cantidad (80\%) que los nacionales que alcanzaron el 20\%. En el año 1998 hubo más colaboraciones nacionales $(73 \%)$ que las internacionales 27\%. En el año 1999 hubo la misma cantidad de trabajos en colaboración nacional (50\%) y internacional 
(50\%). En el año 2000 la cantidad de colaboraciones internacionales fue mayor (67\%) que la nacional (33\%).

Los trabajos en colaboración internacional fueron en su mayoría con España, seguido por Argentina, Cuba y Portugal. En la tabla 4 se detalla el porcentaje de estas colaboraciones..

Tabla 4 Distribución de las cooperaciones internacionales en Brasil

\begin{tabular}{l|l|l}
\hline \multicolumn{1}{c|}{ Países } & Cantidad & \multicolumn{1}{c}{$\%$} \\
\hline Argentina & 1 & 6 \\
\hline Cuba & 1 & 6 \\
\hline España & 12 & 70 \\
\hline Francia & 1 & 6 \\
\hline Portugal & 2 & 12 \\
\hline Total & 17 & 100 \\
\hline
\end{tabular}

En el Gráfico 10 se presenta el índice de coautoría.

\section{Gráfico 10}

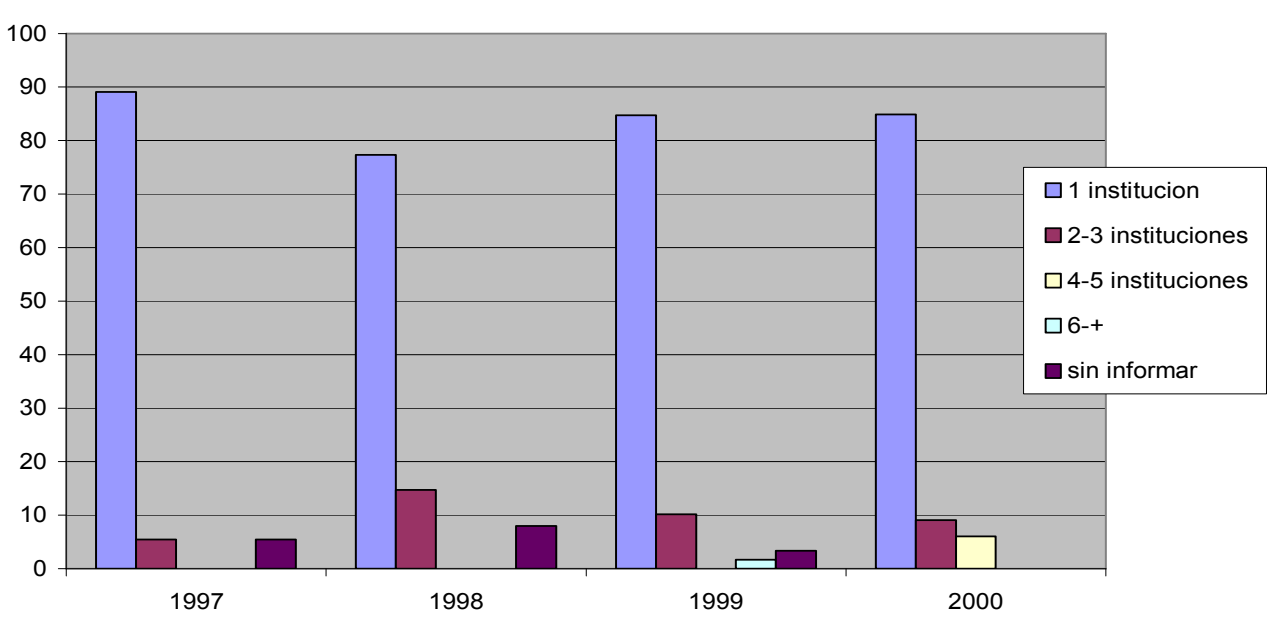

Los resultados presentan que no hubo una tendencia de colaboración en los trabajos de Ciencia Sociales y Humanidades. Los trabajos firmados por una única institución fueron mayoría en los años es- tudiados representando el $90 \%, 77 \%, 85 \%$ y $85 \%$ respectivamente.

Estableciendo una comparación en las colaboraciones registradas en las áreas estudiadas se puede decir que no hay una 
semejanza entre ellas. El área de Ciencia y Tecnología tiene una característica bien acentuada de colaboración, principalmente internacional, en cuanto que el área de Ciencias Sociales y Humanidades mantiene una actuación más individual en la producción de sus trabajos.

\section{CONCLUSIONES}

Los resultados de la investigación presentan que la aportación brasileña al conjunto de las bases ICYT e ISOC es mínima, tal afirmación esta basada en la participación brasileña en dichas bases de datos que en Ciencia y Tecnología no llegó a representar el $1 \%$ y en Ciencia Sociales y Humanidades no alcanzó el $0,50 \%$ de los trabajos recogidos.

La distribución de los trabajos por temática indica que el campo de la Ciencia y Tecnología ocupa el primer lugar, ese resultado es debido por las características de este campo científico que abarca una temática muy amplia, con elevado número de disciplinas y por sus características más universal es de aplicabilidad.

Por otro lado el área de Ciencias Sociales y Humanidades tienen características más regionales o locales, las cuales son factor determinante de su utilidad llevándolas a destinarse al público interno, o sea, su posibilidad de aplicabilidad universal es menor.
El idioma más utilizado en ambas áreas para publicación de los trabajos es el español, ello es lógico, si se observa que las revistas recogidas en las bases ICYT y ISOC son españolas. Hubo artículos publicados en portugués, su representatividad fue mayor en el área de Ciencias Sociales y Humanidades, representa el $16 \%$. En Ciencia y Tecnología representa el $9 \%$. El portugués es uno de los idiomas oficiales de la Comunidad Europea y es el sexto idioma mas hablado en el mundo, representa unos 170 millones de personas, pero los países de habla portuguesa son naciones poco representativas en el escenario de I + D, por el cual, su representación mayor son las áreas de sociales y humanidades. El idioma inglés representa el segundo idioma más utilizado en los trabajos recuperados en el área de Ciencia y Tecnología. El inglés es el idioma más hablado en el mundo científico y de los negocios, se habla en más de 60 países y es el idioma principal del control aéreo, de las conferencias internacionales, de la informática y obviamente de los científicos.

Entre las instituciones la universidad se destaca en ambas áreas, alcanza el $91 \%$ en Ciencias Sociales y Humanidades y el $78 \%$ en Ciencia y Tecnología. El área de Ciencia y Tecnología tuvo varios trabajos de empresas, laboratorios, centros de investigaciones y hospitales entre otros. Tuvo una característica variada en la ori- 
gen del organismo de trabajo de los autores. El área de Ciencias Sociales y Humanidades contó con la participación de universidades, fundaciones y organismos del gobierno, tales como la Fiscalía, La Embajada de Brasil en España o la Oficina Comercial.

La región de mayor concentración de los organismos de trabajo de los autores es la Sudoeste en ambas áreas. Ella alcanza el $67 \%$ en Ciencia y Tecnología y el $57 \%$ en Ciencias Sociales y Humanidades, este resultado confirma estudios similares en el campo de la aportación científica de las regiones brasileñas (TARGINO; GARCIA, 2000). La región Sudoeste concentra el mayor número de investigadores y de cursos de postgrado de Brasil y también es la región de mayor inversión del Gobierno Federal. Por lo expuesto es justificable su posición en el rango de regiones. El segundo lugar lo ocupa la región Sur, alcanza el $24 \%$ en Ciencia y Tecnología y el $32 \%$ en Ciencias Sociales y Humanidades, pero no mantuvo su presencia en todos los años estudiados, comparte con las regiones Nordeste y Centro-Oeste la posición. Este resultado contraria otros estudios, pues la región Nordeste no se presenta con gran cantidad de trabajos en los estudios citados anteriormente. Ella representa el $15 \%$ en Ciencias Sociales y Humanidades y el $19 \%$ en Ciencia y Tecnología. La región Centro-Oeste alcanza el $13 \%$ en Ciencia y
Tecnología y el $11 \%$ en Ciencias Sociales y Humanidades. La región menos representativa es la Norte, no presenta trabajos en Ciencia y Tecnología y en Ciencias Sociales y Humanidades alcanza el 5\%.

Los trabajos en colaboración son más representativos en el área de Ciencia y Tecnología, en las áreas de Ciencias Sociales y Humanidades se caracteriza por trabajos individuales. El comportamiento de la producción es diferente, dependiendo de las áreas científicas que se analicen; y que se requiere buscar indicadores complementarios para tener un mejor análisis nacional y regional.

\section{REFERENCIAS}

BRAUN, T. Scientometric Indicators: a 32Country Comparative Evaluation of Publishing Performance and Citation Impact. Singapur [etc.]: World Scientific, [1985].

BUSHA, C. H. Métodos de investigación en Bibliotecología: técnicas e interpretación. México: Universidad Nacional Autónoma de México, 1990.

CALLON, Michel. Cienciometría: la medición de la actividad científica: de la bibliometría a la vigilancia tecnológica. Gijón: Trea, D.L. 1995.

DIEZ HOYO, M. del C. Balance da producción científica europea de monografías y artículos de revistas sobre América Latina entre 1980 y 1989. REDIAL, n.2, p.7-20, 1993.

EGGHE, L. Introduction to informetrics: quantitative methods in library, documentation and information science. Amsterdam [etc.]: Elsevier Science Publishers, 1990. 
FRANKEL, M. S.; CAVE, J. Evaluating science and scientists. Budapest: Central European University Press, 1997.

GARCÍA ZORITA, J. C. La actividad científica de los economistas españoles, en función del ámbito nacional o internacional de sus publicaciones: estudio comparativo basado en un análisis bibliométrico durante el período 1986-1995. Tesis (Doctorado en Documentación) Departamento de Biblioteconomía y Documentación, Universidad Carlos III de Madrid, Getafe, 2000.

LASCURAIN SÁNCHEZ, M. L. Análisis de la actividad científica y del consumo de información de los psicólogos españoles del ámbito universitario durante el período 1986-1995. Tesis (Doctorado en Documentación) Departamento de Biblioteconomía y Documentación, Universidad Carlos III de Madrid, Getafe, 2001.

LÓPEZ LÓPEZ, P. Introducción a la bibliometría. Valencia: Promolibro, 1996.

MARTÍN MORENO, M. del C. Hábitos y necesidades de la información de los científicos experimentales encuadrados en el área de ciencias de la vida. Tesis (Doctorado en Documentación) Departamento de Biblioteconomía y Documentación, Universidad Carlos III de Madrid, Getafe, 1999.

MARTÍN SEMPERE, M. J.; URDIN CAMINOS, M. C. Producción científica de los países latinoamericanos a través de las revistas españolas en ciencia y tecnología durante el período de 1983-1988. Rev. Esp. Doc. Cient., v. 14, n.2, p.143-153, 1991.

MORILLO ARIZA, F. Estudio de la interdisciplinariedad en la ciencia a través de indicadores bibliométricos. Tesis (Doctorado en Documentación) Departamento de Biblioteconomía y Documentación, Universidad Carlos III de Madrid, Getafe, 2000.

NACIMIENTO, M. J. Producción científica brasileña en España: documentación de las tesis doctórales. Ci. Inf., Brasilia, v. 29, n. 1, p. 3-13, jan./abr., 2000.
SPAGNOLO, F. Brazilian scientist's publications and mainstream science: some policy implications: the case of chemical and electrical engineering. Scientometrics, v. 18, n. 3-4, p. 205-218, 1990.

SPINAK, E. Diccionario enciclopédico de bibliometría, cienciometría e informetría. Caracas: Unesco-CII/I, 1996.

TAGUE-SUTCLIFFE, Jean. Measuring information: an information services perspective. San Diego [etc.]: Academic Press, cop. 1995.

TARGINO, M. G.; GARCIA, J. C. R. Ciência brasileira na base de dados do Institute for Scientific Information (ISI). Ci. Inf., Brasilia, v. 29, n. 1, p. 103-117, jan./abr., 2000.

VALDERRAMA ZURIÁN, J. C. Bibliografía española e internacional de estudios bibliométricos. Valencia: Instituto de Estudios Documentales e Históricos sobre la Ciencia, 1996.

\section{Vanda Ferreira dos Santos \\ Doutoranda em Documentação pela \\ Universidad Carlos III de Madrid santos vanda@hotmail.com}

\section{Title}

Bibliometrics research of Brazilian scientific production in Spanish's journals between 1997 to 2000.

\section{Abstract \\ Bibliometrics research of Brazilian scientific production in Spanish's journals evaluating the Brazilian scientific authors' productions in Spain. The research carried through analysing the scientific papers published in Spanish's journals covered in the ICYT (Technology and Science) and ISOC (Humanities and Social Sciences) data base of Scientific Superior Council Investigation (CSIC). The results shows that the Brazilian contribution in the data bases aren't representative: in Technology and Science it's $1 \%$ and in Humanities and Social Sciences it's 0,50\%.}




\section{Keywords}

Bibliometrics; Scientific Production; Brazilian

Scientists;

\section{Resumen}

Estudio bibliometrico sobre la producción científica brasileña en revistas españolas con el objetivo de evaluar la producción científica de autores brasileños en España. El análisis ha sido realizado a través de los artículos científicos publicados en revistas españolas recogidas en las bases de datos de Ciencia y Tecnología (ICYT) y de Ciencias Sociales y Humanidades (ISOC) del Consejo Superior de Investigaciones Científicas (CSIC). Los resultados de la investigación presentan que la aportación brasileña al conjunto de las bases ICYT e ISOC es mínima, tal afirmación esta basada en la participación brasileña en dichas bases de datos que en Ciencia y Tecnología no llegó a representar el $1 \%$ y en Ciencia Sociales y Humanidades no alcanzó el $0,50 \%$ de los trabajos recogidos.

\section{Palabras Clave}

Bibliometria; Producción Científica; Revistas Españolas

Artigo recebido em: 23/09/2002 